\title{
Contrastivism and Non-Contrastivism in Scientific Explanation
}

Yafeng Shan

The Cohn Institute for the History and Philosophy of Science and Ideas, Tel Aviv

University, Tel Aviv, Israel

ys1@mail.tau.ac.il

\begin{abstract}
The nature of scientific explanation is controversial. Some maintain that all scientific explanations have to be contrastive in nature. (Contrastivism) However, others argue that no scientific explanation is genuinely contrastive. (Non-contrastivism) In addition, a compatibilist view is developed. It is argued that the debate between contrastivism and non-contrastivism is merely a linguistic dispute rather than a genuine disagreement on the nature of scientific explanation. Scientific explanations are both contrastive and non-contrastive in some sense. (Compatibilism) This paper examines the debate between contrastivism and non-contrastivism in scientific explanation. It begins with a critical review of the arguments for contrastivism, noncontrastivism, and compatibilism, and concludes with some remarks on the prospect of the issue.
\end{abstract}

\section{Key Words}

Scientific explanation; contrastive explanation; non-contrastive explanation; contrastivism; non-contrastivism; compatibilism; why-question

\section{Acknowledgements}

This work is supported by the Humanities Fund of the Israeli Council of Higher Education and Israel Science Foundation Grant (1128/15). In addition, I would like to thank two anonymous reviewers for the helpful comments. 


\section{Introduction}

Most philosophers and scientists ${ }^{1}$ may admit that one of the most important tasks for scientists is to answer why-questions. In other words, science is not only about description but also about explanation ${ }^{2}$. However, the nature of scientific explanation is controversial. Some (e.g., Botterill, 2010; Garfinkel, 1981; Khalifa, 2010; van Fraassen, 1980) contend that all scientific explanations have to be contrastive in nature. (Contrastivism) In other words, what gets explained is in fact of the form "why $p$ rather than $q$ " rather than simply "why p." As Bas van Fraassen (1980, p. 127) famously argues, "The correct general, underlying structure of a why-question is ... Why (is it the case that) $P$ in contrast to (other members of) $X$ where $X$, the contrast class, is a set of alternatives." However, others (e.g., Carroll, 1997; Ruben, 1987; Strevens, 2008; Temple, 1988) argue that no scientific explanation is genuinely contrastive. All contrastive explanations are always reducible to non-contrastive explanations. (Non-contrastivism) ${ }^{3}$ In addition, a compatibilist view has been developed recently (e.g., Gijsbers, 2018). It is argued that the debate between contrastivism and non-contrastivism is merely a linguistic dispute rather than a genuine disagreement on the nature of scientific explanation. Scientific explanations are both contrastive and non-contrastive to some extent. (Compatiblism) This paper examines the debate between contrastivism and non-contrastivism in scientific explanation. Section 2 revisits the arguments for contrastivism. Section 3 reviews the arguments for non-contrastivism. Section 4 discusses the proposal of compatiblism. Section 5 makes some general remarks.

\section{Contrastivism}

The argument for constrastivism is deeply rooted in a conviction, which is well summarised by George Botterill (2010, p. 288): "explanations... are contrastive; and that even when no contrast is made explicit in an explanatory inquiry they are still implicitly contrastive." Such a conviction arises from a concern on the ambiguity of the question of the form "why $p$ ". Consider the question

(Q) Why did this sample of copper burn green?

It is obvious that $(\mathrm{Q})$ itself is quite ambiguous. It might mean completely different questions as follows.

(Q1) Why did this sample of copper burn green rather than yellow?

(Q2) Why did this sample of copper burn green rather than not burn?

(Q3) Why did this sample of copper rather than other samples burn?

Clearly, (Q1), (Q2), and (Q3) must be answered differently. Accordingly, what counts as a satisfactory answer to (Q) depends on which of (Q1), (Q2), and (Q3) was meant. Thus, it is argued that there is no satisfactory answer to questions like (Q) unless it is explicated clearly in a contrastive way. In other words, all non-contrastive explanations are just seemingly non-contrastive.

An important implication of this is that all seemingly non-contrastive explanations are reducible to contrastive explanations. This seems to be problematic for many. Michael Strevens (2008, p. 177), for example, argues that the reformulation of "why $p$ " as 
"why $p$ rather than $\neg p$ " leads to a rejection of constrastivism. His argument is technically sophisticated, but the basic idea is as follows. ${ }^{4}$

P1. All scientific explanations are contrastive.

$\mathrm{P} 2$. From $\mathrm{P} 1$, it is deducible that a seemingly non-contrastive scientific explanation of an event $p$ would have been interpreted as an explanation of why $p$ rather than $\neg p .^{5}$

P3. The explanation of why $p$ rather than $\neg p$ requires an explanation of the nonoccurrence of $\neg p$.

$\mathrm{P} 4$. The explanation of why $p$ does not require an explanation of the non-occurrence of $\neg p$.

C. $\mathrm{P} 2$ is false, and $\mathrm{P} 1$ is thus false.

At first glance, Streven's argument is not decisive. The contrastivist may respond that $\mathrm{P} 2$ is not true. A contrast of $p$ is not necessarily $\neg p$. Thus, $\mathrm{P} 2$ should be revised as

P2': From P1, it is deducible that a seemingly non-contrastive scientific explanation of an event $p$ would have been interpreted as an explanation of why $p$ rather than $c$ where $c$ is not identical with $p$.

Accordingly, the argument is thus reformulated as follows.

P1. All scientific explanations are contrastive.

$\mathrm{P} 2$ '. From $\mathrm{P} 1$, it is deducible that a seemingly non-contrastive scientific explanation of an event $p$ would have been interpreted as an explanation of why $p$ rather than $c$ where $c$ is not identical with $p$.

P3'. The explanation of why $p$ rather than $c$ requires an explanation of the nonoccurrence of $c$.

P4'. The explanation of why $p$ does not require an explanation of the non-occurrence of $c$.

$\mathrm{C}^{\prime} . \mathrm{P} 2$ ' is false, and $\mathrm{P} 1$ is thus false.

Now what is at issue is whether P4' is true. Simply put, is there any explanation which does not involve any contrasts? Following Paul Humphreys's suggestion (1989), Paul Markwick (1999) argues that there are some non-contrastive explanations which cannot be analysed in terms of contrasts. He explicates the question of "why this sample of copper burnt green" in the following way.

[W] hat could need explaining is simply the green colour of the event; one might want to know why the event had exactly this property without wondering, for example, why it was green rather than red. (Markwick, 1999, p. 195)

In short, what is to be explained is just why there was a green flame. In other words, what is to be explained is the cause or the mechanism of a green flame. Why it is not a yellow flame instead is not the concern. For the contrastivist, a standard response might be that the non-contrastive explanandum that there is a green flame can be reformulated as a contrastive one as follows. It is the case that there is a green flame rather than it not being the case that there is a green flame. However, such a response is unsatisfactory. Reading "why $p$ " as "why $p$ rather than $\neg p$ " seems to be an ad hoc move. As Victor Gijsbers (2018, p. 1222) points out, it is "only undertaken to save the contrastive view but cannot be otherwise justified." What is worse, reading "why $p$ " as "why $p$ rather than $\neg p$ " leads to a rejection of contrastivism, as Strevens argues. 


\section{Non-contrastivism}

The main strategy of defending non-contrastivism is to show that all contrastive explanations are reducible to non-contrastive explanations. In general, there are three lines of argument for the view that contrastive explanations are reducible to noncontrastive explanations. It can be argued that the contrastive explanation "Why $p$ rather than $q$ " can be simply interpreted as a conjunctive explanation of "why $p$ and not q." (Carroll, 1997; Temple, 1988) If so, then all the contrastive explanations are reducible to the non-contrastive explanations. It can also be argued that to explain why $p$ rather than $q$ is to explain why $p$ and why $p$ eclipses $q$. (Ruben, 1987) A third way is to argue that to explain why $p$ rather than $q$ is to explain why $p$ given that either $p$ or $q$. (Hitchcock, 1999) However, none of the three lines is fully satisfactory.

It is clear that explaining why $p$ rather than $q$ is not identical with explaining why $p$ and not $q$. In the cases where $p$ and $q$ are incompatible with each other, to explain why $p$ and not $q$ is logically equivalent to explain why $p$, as $p$ and not $q$ is logically equivalent to $p$. For example, explaining why Donald Trump rather than Hillary Clinton became president of the USA in 2016 is tantamount to explaining why Donald Trump became president of the USA in 2016, as there is only one president of the USA. Thus, in these cases, the conjunction of the explanations of why $p$ and of why not $q$ is no more than the explanation of why $p$, from a logical point of view. However, it is obvious that the contrastive explanation of why $p$ rather than $q$ is not. In the case of USA presidential election, we know that the fact that Donald Trump became president of the USAeclipses the fact that Clinton became president of the USA. We may be happy with that Trump won the majority of the electoral votes explains why Trump became president of the USA. We may be also happy with that that Trump won the majority of the electoral votes explains why Trump became president of the USA and that why Clinton failed to become president of the USA. But we would be definitely unsatisfied with that Trump won the majority of the electoral votes explains why Trump rather than Clinton became president of the USA, as we are interested in why it is Trump rather than Clinton who won the majority of the electoral votes. An adequate explanation of why Donald Trump rather than Hillary Clinton became president of the USA in 2016 has to encompass the explanans concerning both Trump and Clinton. It is in this sense that the explanation of why Donald Trump rather than Hillary Clinton became president of the USA in 2016 is not identical with the explanation of why Donald Trump became president of the USA in 2016. Thus, in the cases where $p$ and $q$ are incompatible with each other, explaining why $p$ rather than $q$ is not identical with explaining why $p$ and not $q$. In other words, not all the contrastive explanations of why $p$ rather than $q$ can be reformulated as a conjunction of the explanation of why $p$ and the explanation of why not $q$.

It is also clear that to explain why $p$ rather than $q$ is not identical with to explain why $p$ and why $p$ eclipses $q$. For example, it is sufficient to explain why Jones rather Smith contracted paresis by pointing out that Jones had untreated syphilis, as syphilis is causally necessary for paresis. However, it is not necessary to explain why Jones's affliction eclipses Smith's in order to explain why Jones rather than Smith contracted paresis. As Peter Lipton (1987, p. 208) indicates, "Jones's affliction hardly protects Smith." In other words, in order to explain why $p$ rather than $q$, we do not always expect an explanation of why $p$ eclipses $q$, especially in the cases where $p$ and $q$ are compatible. 
Compared with the first two lines of argument, it seems more promising to argue that to explain why $p$ rather than $q$ is to explain why $p$ given that either $p$ or $q$. A contrastive explanation can be well reformulated as a non-contrastive explanation with a fixed background. Nevertheless, it is doubtful that a non-contrastive explanation with a fixed background is still non-contrastive in nature. What the contrastivist tries to emphasise is that all scientific explanations are relative to some extent. As Lipton (2004, p. 33) explicates,

A contrastive phenomenon consists of a fact and a foil, and the same fact may have several different foils. We may not explain why the leaves turn yellow in November simpliciter, but only for example why they turn yellow in November rather than in January, or why they turn yellow in November than blue.

Thus, the contrastivist may accept that every contrastive explanation can be reformulated as a non-contrastive explanation with a presupposition that limits the explanatory space, but still insist that all scientific explanations are contrastive in this sense. It is not clear, however, whether all the non-contrastivists would be happy to accept this.

\section{Compatibilism}

Gijsbers (2018) has recently developed a compatibilist thesis by arguing that a contrastive explanation is in fact a partial explanation of a non-contrastive explanandum with respect to a particular and limited explanatory space, while a nonconstrastive explanation is a full explanation of all the possible constrastive explananda. Therefore, the tension between contrastivism and non-contrastivism can be reconciled by arguing that contrastiveness and non-contrastiveness only reflect the different aspects of scientific explanation. The debate between contrastivism and non-contrastivism is merely a linguistic dispute rather than a disagreement on the nature of scientific explanation.

Gijsbers' proposal is prima facie promising, since it well explains away the disagreement between contrastivists and non-contrastivists. For Gijsbers, there are some merits in both contrastivism and non-contrastivism. The reason that contrastivism is appealing to many is that it highlights the relativity (or incompleteness) of scientific explanation. Scientific explanations are always relative, or partial to some extent. As many (e.g., Hempel, 1965, pp. 421-423; Lipton, 2004, p. 33; van Fraassen, 1980, p. 128) indicate, we do not explain events, only aspects of events. For example, we do not explain the eclipse tout court, but why it lasted as long as it did, or why it was not visible from a certain place. On the other hand, the idea of non-contrastivism is plausible, because scientists are often concerned with non-contrastive explananda. For example, though why sea temperature is rising rather than not might be an interesting scientific question, climate scientists may only be interested in the question of why sea temperature is rising. ${ }^{6}$ Gijsbers' thesis well accommodates these motivations by arguing that all contrastive explanations are in fact partial explanations of some non-contrastive explananda, while all actual noncontrastive explanations only account for a limited set of contrasts.

However, one might wonder whether the distinction between contrastiveness and noncontrastiveness is still necessary to hold if compatibilism is accepted. On the one hand, if a non-contrastive explanation is a full explanation of all the possible 
contrastive explananda, it seems that we can never have a non-contrastive explanation. As Gijsbers (2018, p. 1224) himself recognises, "no actually given explanation is ever the explanation of a non-contrastive explanandum." If so, the concept of contrastive explanation seems to be empty. On the other hand, if a contrastive explanation is a partial explanation of a non-contrastive explanandum with respect to a particular and limited explanatory space, we do not have any contrastive explanation either. When looking at our contrastive explanation closer, we may find the structure of their explananda is not simply in form of "why $p$ rather than $q$ ?" Rather it is much more complicated, involving more contrasts. As Gijsbers argues, we usually want to explain a doubly contrastive fact. Suppose that one looks out of the window of my third floor office, and sees her colleague Peter falling past it. Rather than to wonder "why Peter is falling past my window rather than being safely inside", one usually looks for the explanations such as "why Peter's attitude towards the medical establishment (rather than other possible attitudes) caused him to fall past my window (rather than being safely inside", or "why the non-existence of benevolent and activist angels (rather than their existence) caused Peter to fall past my window (rather than being safely inside)." Still, all these multiply contrastive explanations can be reformulated in terms of contrastive explananda. For example, a doubly contrastive explanandum can be characterised as why $p$ rather than $q$ is predicated on $x$ rather than $y$. Nevertheless, it is really doubtful that the distinction between contrastive and non-contrastive explanations is useful or beneficial to analyse the nature of scientific explanation if all actual scientific explanations have to be analysed with multiple contrasts.

\section{Discussion}

From a logical point of view, there are five possibilities of the relation of contrastive explanations and non-contrastive explanations in science. (1) Scientific explanations are either contrastive or non-contrastive. (2) All scientific explanations are contrastive in nature, though there are some seemingly non-contrastive explanations. (3) All scientific explanations are non-contrastive in nature, though there are some seemingly contrastive explanations. (4) There are three types of scientific explanation: contrastive explanation, non-contrastive explanation, and both contrastive and noncontrastive explanation. (5) All scientific explanations are both contrastive and noncontrastive. Clearly, the contrastivist aims at defending (2), while the noncontrastivist argues for (3). However, neither succeeds.

The strategy of the defence of non-contrastivism is incomplete. Even if it is successfully shown that all contrastive explanations are reducible to non-contrastive explanations, it does not imply that all scientific explanations are non-contrastive in nature. In order to defend the stance that all scientific explanations are noncontrastive, one has to show that non-contrastive explanations are more fundamental than contrastive explanations in the sense that (a) there is a genuine distinction between contrastive and non-contrastive explanations, and (b) all contrastive explanations are reducible to non-contrastive explanations. Without a justification of (a), the possibility cannot be excluded that contrastive and non-contrastive explanations are reducible to each other. If so, the distinction between contrastive and non-contrastive explanations is just a matter of convention. Thus, the claim that all scientific explanations are contrastive and the claim that all scientific explanations are non-contrastive can be both true. In other words, non-constrastivism cannot be successfully defended unless (a) and (b) are both justified. In a similar vein, the 
defence of contrastivism is incomplete, too. Arguing that all non-contrastive explanations are contrastive is insufficient. It has also to be shown that there are some contrastive explanations which are not identical with or reducible to noncontrastive explanations.

The compatibilist proposal shows (5) by arguing that all scientific explanations are both contrastive and non-contrastive in the sense that the distinction between contrastive and non-contrastive explanations is merely a linguistic matter. However, such a proposal undermines the core of the debate to some extent. An important consequence is that neither contrastiveness nor non-contrastiveness captures the very nature of scientific explanation. Of course the debate between contrastivism and noncontrastivesm is still meaningful in a pragmatic sense. It is still debatable whether a contrastive analysis of scientific explanation is better than a non-contrastive one. For example, Petri Ylikoski (2007) argues that contrastivism should be interpreted as "all explananda can be analysed as contrastive" and a contrastive approach is more fruitful than a non-contrastive one. Another important lesson from the compatibilist proposal is that it might be worth trying a completely different approach to the relative (or incomplete) nature of scientific explanation, given the insignificant distinction between contrastiveness and non-contrastiveness. (We may need a new theory of explanatory relativity which is not in terms of contrasts!)

\section{Bibliography}

Botterill, G. (2010). Two Kinds of Causal Explanation. Theoria, 76, 287-313.

Carroll, J. W. (1997). Lipton on compatible contrasts. Analysis, 57(3), 170-178. https://doi.org/10.1093/analys/57.3.170

Duhem, P. M. M. (1954). The Aim and Structure of Physical Theory. Princeton, NJ: Princeton University Press.

Garfinkel, A. (1981). Forms of explanation: Rethinking the questions in social theory. New Haven: Yale University Press.

Gijsbers, V. (2018). Reconciling Contrastive and Non-contrastive Explanation. Erkenntnis, 83(6), 1213-1227. https://doi.org/10.1007/s10670-017-9937-8

Hempel, C. G. (1965). Aspects of Scientific Explanation and Other Essays in the Philosophy of Science. New York: The Free Press.

Hitchcock, C. (1999). Contrastive explanation and the demons of determinism. British Journal for the Philosophy of Science, 50(4), 585-612.

https://doi.org/10.1093/bjps/50.4.585

Humphreys, P. (1989). The chances of explanation: Causal explanation in the social, medical, and physical sciences. Princeton, NJ: Princeton University Press.

Khalifa, K. (2010). Contrastive Explanations as Social Accounts. Social Epistemology, 24(4), 263-284.

Lipton, P. (1987). A Real Contrast. Analysis, 47(4), 207-208.

Lipton, P. (2004). Inference to the Best Explanation (2nd ed.). London and New York: Routledge. 
Markwick, P. (1999). Interrogatives and Contrasts in Explanation Theory. Philosophical Studies, 96(2), 183-204. Retrieved from http://www.jstor.org/stable/4320980

Pearson, K. (1900). The Grammar of Science (2nd ed.). London: Adam and Charles Black.

Ruben, D.-H. (1987). Explaining contrastive facts. Analysis, 47(1), 35-37. https://doi.org/10.1093/analys/47.1.35

Scriven, M. (1962). Explanation, Predictions, and Laws. In H. Feigl \& G. Maxwell (Eds.), Scientific Explanation, Space and Time (Vol. 3, pp. 170-230). Minneapolis, MN: University of Minnesota Press.

Strevens, M. (2008). Depth: An account of scientific explanation. Cambridge, MA: Harvard University Press.

Temple, D. (1988). The Contrast Theory of Why-Questions. Philosophy of Science, $55(1), 141-151$.

van Fraassen, B. C. (1980). The Scientific Image. Oxford: Clarendon Press.

Ylikoski, P. (2007). The Idea of Contrastive Explanandum. In J. Persson \& P. Ylikoski (Eds.), Rethinking Explanation (pp. 27-42). Dordrecht: Springer.
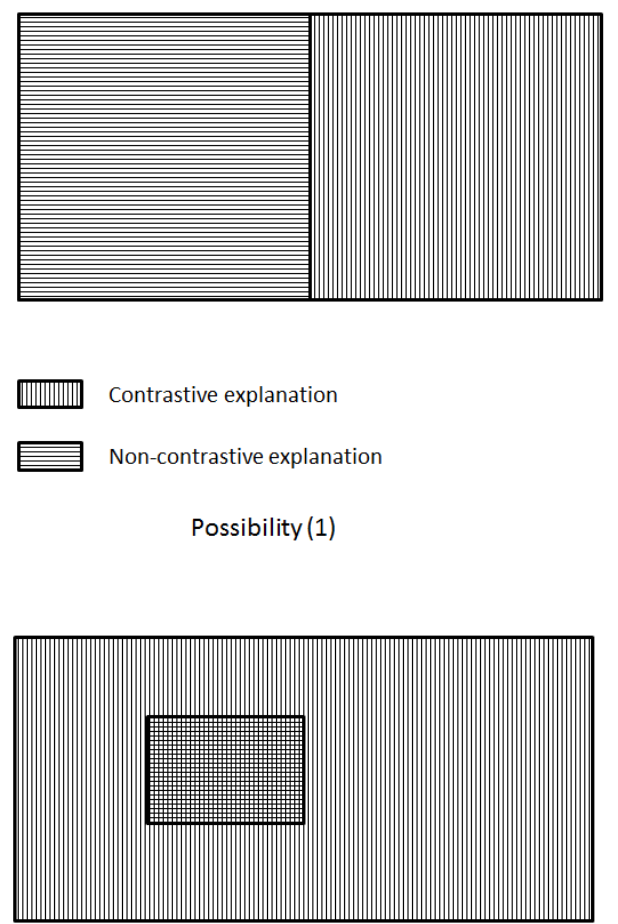

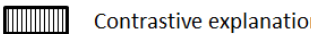

Non-contrastive explanation

Possibility (2) 


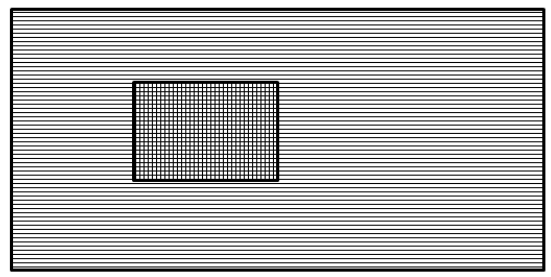

|

Non-contrastive explanation

Possibility (3)

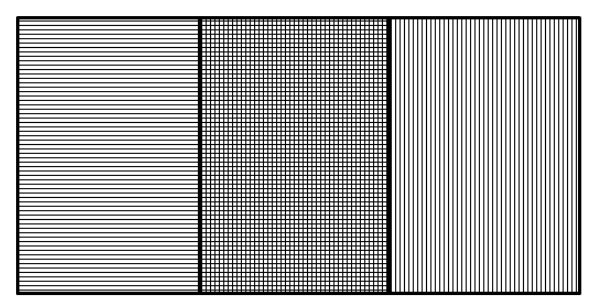

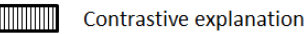

Non-contrastive explanation

Possibility (4)

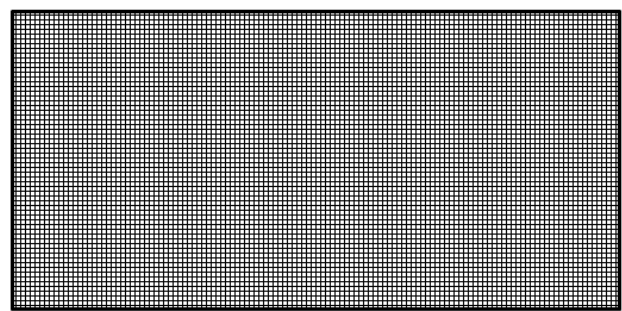

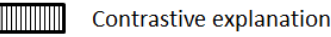

Non-contrastive explanation

Possibility (5)

\footnotetext{
1 Pierre Duhem (1954) and Karl Pearson (1900) are two famous exceptions.

2 It has been widely received among philosophers that scientific explanations are answers to why-questions. However, some (e.g., Botterill, 2010; Scriven, 1962) argue that not all scientific explanations are the answers to why-questions. Quite a few explanations are in fact the answers to how-questions such as "How can a neutrino be detected, when it has zero mass and zero charge?" I am not committed to either view here. In this paper, I focus on the scientific explanations as the answers to why-questions.

${ }^{3}$ The debate between contrastivism and non-contrastivism is traditionally applicable to explanation in general. However, I shall focus on scientific explanation here. Thus, I shall avoid using the famous examples like the robber Willy Sutton, as they might not be appropriate for my purpose.

${ }^{4}$ For a detailed technical discussion, see Strevens (2008, pp. 174-177).

${ }^{5}$ For Strevens, $p$ and $\neg p$ here refer to events.
} 
${ }^{6} \mathrm{~A}$ crucial difference is that when scientists ask "why sea temperatures are rising" , they are typically looking for the causes of the rise of sea temperature. Once they are certain about a factor, say F, that causes the rise of sea temperature, they have an answer to the question. It is different from the answer to "why sea temperatures are rising rather than not." In addition to show that $\mathrm{F}$ causes the rise of sea temperature, the scientists have to show why $\mathrm{F}$ eclipses another factor F' , which causes the fall of sea temperature, in order to have an adequate explanation of why sea temperatures are rising rather than not. 